\title{
Taming Cytokine Storm Syndrome (CSS) of COVID 19 infection: Are we far from reaching the finishing line?
}

\author{
Rumi Ahmed Khan
}

Cytokines are small secreted proteins released by cells have a specific effect on the interactions and communications between cells. Cytokines are made by many cell populations, but the predominant producers are helper T cells (Th) and macrophages. Cytokine is a general name; other names include lymphokine (cytokines made by lymphocytes), monokine (cytokines made by monocytes), chemokine (cytokines with chemotactic activities), and interleukin (cytokines made by one leukocyte and acting on other leukocytes). ${ }^{1}$

Cytokine Storm Syndrome (CSS), also occasionally known as Cytokine Release Syndrome (CRS), is a clinical syndrome that results from systemic inflammatory response to varying pathway of cytokine activation in response to a diverse group of insults ${ }^{2}$.In this specific clinical state, the cells of the immune system are overly activated and produce large amounts of cytokines, which in turn, generates a hyperinflammatory phenomenon. Various inflammatory cytokines or chemokines such as tumor necrosis factor (TNF)- $\alpha$, type I and II interferons (IFNs), interleukin (IL)-1, IL-6, CCL2, or monocyte chemotactic protein-1 (MCP-1), as well as immunosuppressive cytokines such as IL-10 or transforming growth factor- $\beta$, various immune cells such as T cells, B cells, dendritic cells (DCs), macrophages have been implicated as components of cytokine storm ${ }^{3}$.

While discussing CSS, CRS, we also need to put another condition in the same spectrum. Hemophagocytic lymphohistiocytosis (HLH), is a severe, life-threatening inflammatory syndrome associated with intense cytokine release $^{4}$. In HLH, aberrant activation of T cells, natural killer (NK) cells, and macrophages causes overproduction of inflammatory cytokines (i.e., cytokine storm) and hemophagocytosis. Many viral diseases have been documented to cause HLS, i.e. Epstein-Barr virus (EBV) and herpes simplex virus (HSV) infections have been abundantly documented to be triggers of HLH. HLH has also been observed with other viruses like cytomegalovirus, hepatitis A, parvovirus B19, adenovirus, influenza, as well as different bacteria, fungi, parasites. The commonly laboratory findings of HLH have been commonly reported as thrombocytopenia, coagulation disorders, while hypofibrinogenemia and raised D-dimer levels, abnormal alkaline phosphatase/ transaminase), increased serum lactate dehydrogenase $(\mathrm{LDH})$. Elevated C-reactive protein (CRP) concentration is a common laboratory anomaly as is increased ferritin concentrations which is seen in $90-100 \%$ of adult manifesting HLH. The source of ferritin is increased secretion of ferritin by macrophages or hepatocytes ${ }^{5}$. Clinicians managing patients with CoViD-19 will notice that most of the

above-mentioned laboratory abnormalities e.g. elevated CRP, Ferritin, transaminases, LDH, LDH and lymphopenia are also frequently observed in their patients with Covid-19 going through what is clinically suspected as CSS. 'Macrophage Activation Syndrome' (MAS) also may be a syndrome identical to "cytokine storm" as a common end point of multiple different disease pathogenesis. As a contributing immune pathway in CSS, activation of macrophages has been particularly studied and, in many instances, 'macrophage activation syndrome' (MAS) has been used synonymously with CSS or a part of CSS spectrum condition ${ }^{6,7}$. The clinical syndromes related CSS, CRS, Hemophagocytic lymphohistiocytosis (HLH) and Macrophage Activation Syndrome have been discussed either as spectrum of hyperinflammatory syndrome or alterative / different terms used to describe same clinical phenomenon.

Like some other previous novel viral pandemic, during this CoViD-19 pandemic, CSS is back to our attention again. A positive impact of this renewed focus on cytokine storm is that it encourages medical science to invest more time, effort and research focus on understanding this phenomenon. And at the same time the physician community gets reminded that many non-related diseases process they deal with regularly or rarely are in fact the same pathophysiologic derangement started on by a varying causative agent! It probably will not be too radical a statement if we say most, if not all, severe disease processes at some point of its clinical course goes through its own cytokine storm. As much we are aware of CoViD-19 related cytokine storm, we need to be equally aware that many other distinct but common clinical syndromes like Dengue Shock syndrome, acute pancreatitis, fulminant malaria infection, any sort of severe sepsis - all may be cytokine Storm Syndromes under different names.

Without specifying the term cytokine storm, our first recognition of similar clinical syndrome probably came in the form of our recognition of sepsis. Sepsis indeed is the most encountered cytokine storm. In 1904, Sir William Osler in in his book, The Evolution of Modern Medicine paved the way for our present day understanding that sepsis related clinical syndrome are not direct result of the pathogen, but rather the body's immune response to the pathogen ${ }^{3}$.

The term "cytokine storm" seems to be first used in 1993 to describe graft-versus-host disease ${ }^{8}$, .

However, beginning in early 2000, cytokine storm related clinical syndrome started getting more recognizable in infectious diseases, esp. viral infection settings. Many recent reviews have addressed potential cellular and molecular mechanisms contributing to the cytokine storm in viral disease $^{10,11}$, There has been reports of cytokine storm in the 
setting of cytomegalovirus infection, group A streptococcus, influenza virus, variola virus, and the first severe acute respiratory syndrome coronavirus (SARS-CoV). The 2012 H5N1 (Bird Flu) scare and a handful of H5N1 death first brought CSS in mainstream media ${ }^{8}$.

The above discussions point out very clearly that cytokine storm is not one distinct disease entity. It is rather a common clinical end point of different initial insults, be it infectious, be it autoimmune/ inflammatory or be it iatrogenic. Even within each category of causation, the pathogenesis and organ involvement are different based on organism or type of insult. It is not only that cytokine storm is the culmination of a multitude of varying signal transduction pathways depending on the primary source, in recent years there is a clearer understanding of compartmentalization of tissue-specific microenvironments where the processes of cytokine storm begins $^{8}$. Dengue virus related CSS perhaps explains this compartmentalization most clearly. The tissue compartment where dengue related cytokine storm, which we know as Dengue shock syndrome, takes place is the capillary planes resulting in increased capillary permeability and excessive plasma leakage ${ }^{12,13}$. Influenza viruses infect and destroy the ciliated epithelial cells of the conducting airways, whereas SARS-CoV infects type II pneumocytes in the alveolar walls and hantavirus particles infect microvascular endothelial cells in the alveolar walls. These different agents cause localized cytokine storm involving varying cytokine profile but frequently lead to indistinguishable clinical syndromes of acute lung injury (ALI) with respiratory failure? ${ }^{9}$. When these local inflammations or enhanced cytokine activities spill over into the systemic circulation, a systemic process becomes manifest. The systemic process may include persistent hypotension, hyper- or hypothermia, leukocytosis or leukopenia, and thrombocytopenia ${ }^{14}$, or other features we encounter in severe sepsis. Perhaps this systemic process, resulting from spillover of localized process, is CSS as we understand it. And this understanding of cytokine storm also helps us understand why some patients with certain infection, e.g. CoViD-19 gets only minimal upper airway symptoms and gets better while a smaller minority develops high unremitting fever and ARDS.

As we understand that the signal transduction activity leading to cytokine storm originates in the different affected tissue, that measurement of serological biomarkers spilled over from effected tissues to the peripheral blood sleep may not give us a reliable information of the ongoing cytokine storm ${ }^{15,16}$. Hence, we do not have a troponin like instant diagnosis biomarker for Sepsis or IL-6 level may suggestive but not diagnostic of severe CoViD-19 related cytokine storm.

Once viral RNA enters a cell, the host's innate immune system initiates a rapid anti-viral signaling cascade, leading to the production of various cytokines by specific infected cell and innate immune cells. With better understanding of infection/ insult specific varying roles of individual cytokines, the concept of the cytokine storm has become more complicated. Beyond the direct effects of these cytokines on different cell types, their cross-regulatory functions within the cytokine network can have important effects on the outcome of an infection. One useful framework for considering the role of cytokines is to divide them by those that are directly induced by virus infection (primary cytokines) and those that are induced downstream by other cytokines or features of the immune response (secondary cytokines) ${ }^{17}$.

Unprecedented volume of work is going on regarding SARS-CoV-2 immunology related to CSS. As we are early in this disease outbreak, a clear and consensus picture of SARS-CoV-2 immunology is not there yet. What we know so far is that after entering respiratory epithelial cells, SARS-CoV-2 provokes an immune response with inflammatory cytokine production accompanied by a weak interferon (IFN) response ${ }^{18}$. The pro-inflammatory immune responses of pathogenic Th1 cells and intermediate CD14, CD16 monocytes are mediated by membrane-bound immune receptors and downstream signaling pathways. This is followed by the infiltration of macrophages and neutrophils into the lung tissue, which, in some patients, results in a cytokine storm $^{19}$. The virus rapidly activates pathogenic Th1 cells to secrete pro-inflammatory cytokines, such as granulocyte-macrophage colony-stimulating factor (GM-CSF) and interleukin-6 (IL-6). GM-CSF, in turn, further activates CD14, CD16 inflammatory monocytes to produce large quantities of IL-6, tumor necrosis factor-alpha ${ }^{20}$. Membrane-bound immune receptors like Fc and Toll-like receptors may also contribute to an imbalanced inflammatory response, and weak IFN-Alpha induction may be an important amplifier of cytokine production ${ }^{19}$. Neutrophil extracellular traps (NETs), the extracellular nets released by neutrophils, has also been shown to contribute to excessive cytokine release $\mathrm{e}^{21}$.

The cytokine storm in COVID-19 has been shown to take place in its unique tissue plains ${ }^{2}$. Some remarkable autopsy findings revealed that the lymphoid tissues and organs had been destroyed in COVID-19 patients, which is very unusual from CSS in sepsis and CAR T-cell therapy related $\mathrm{CSS}^{22}$.

The quest to treat cytokine storm by immunomodulation is an old one ${ }^{3}$. Sepsis being the most encountered cytokine storm and with identification of tumor necrosis factor (TNF) and interleukin-1 $\beta$ (IL-1 $\beta$ ) as major inflammatory cytokines in sepsis models, serious efforts were made to develop therapeutics by blocking these specific cytokines. However, all such attempts at treating sepsis by inhibiting cytokine signaling pathways failed to yield any positive result $t^{23}$.

A variety of anti-inflammatory drugs including corticosteroids, aspirin, monoclonal antibodies (MAbs), anti-cytokine and anti-chemokine agents, plasma exchange, and statins were unsuccessfully tried to dampen cytokine storm $^{9,24}$.

The failure of therapeutic interventions to curb cytokine storm $^{25,26}$ of sepsis and other syndromes bring home the point that has been elaborately discussed above - cytokine storm is a pathogenesis process that involves a varied spectrum of signal transduction molecules working in different tissue plains. Tissues involved and cytokines recruited - all depend 
on the source of the insult. We have explained that while influenza virus work on one kind of airway cell, SARS-CoV-2 works elsewhere, and Dengue virus works in capillaries - all initiating cytokine storm in different tissues involving a different permutation -combination of cytokines. Hence one size fit all immunomodulation repeatedly failed in clinical trials. While our five decade long quests for a specific randomized controlled trial proven therapeutic intervention in sepsis have been a sad tale of perpetual failure, in only eight months of existence of Covid-19, we now have at least two randomized controlled trials ${ }^{27,28}$ showing mortality benefit of a therapeutic intervention with dexamethasone in patients with moderate to severe Covid-19 going through CSS. One explanation on why decades long quest for a therapeutic agent for cytokine storm of sepsis has been a failure but search for a therapeutic intervention against Covid-19 cytokine storm has been successful is that unlike SARS-CoV-2 induced CoVid-19 related cytokine storm, sepsis is a big basket heterogenous diagnosis caused by a diverse group of causative organisms including bacteria, virus etc.

By the time, this pandemic will be under control, the novel SARS-CoV-2 is tamed, there may well be over 3-4 million dead and millions more suffering from chronic post covid-19 symptoms. Yet positive side of the story is that this pandemic unleashed an unprecedented flurry of scientific activity which not only helped us understand Covid-19 better, these scientific activities gave the humankind a better understanding of medicine as a whole. Thanks to our quest to fight CoViD-19, we'll be better prepared next year not only to treat CoViD-19, we'll be prepared to understand and treat many other ailments those goes through this common clinical pathway known as the CSS

\section{Rani A Khau MD}

Rumi Ahmed Khan, MD. FCCP.

Fellowship Program Director

Orlando Health Pulmonary Critical Care Fellowship

Director

Cardiopulmonary Exercise and Pulmonary Function Laboratory Associate Professor

University of Central Florida College of Medicine, USA

E-mail : rumi.ahmed@gmail.com

\section{References:}

1. Zhang JM, An J. Cytokines, inflammation, and pain. Int Anesthesiol Clin. 2007;45(2):27-37. doi:10.1097/AIA.0b013e318034194

2. Gao YM, Xu G, Wang B, Liu BC. Cytokine storm syndrome in coronavirus disease 2019: A narrative review [published online ahead of print, 2020 Jul 22]. $J$ Intern Med. 2020;10.1111/joim.13144. doi:10.1111/joim.13144

3. Behrens EM, Koretzky GA. Review: cytokine storm syndrome: looking toward the precision medicine era. Arthritis Rheumatol. 2017;69(6):1135-1143. doi: 10.1002/art.40071.

4. Opoka-Winiarska V, Grywalska E, Roliński J. Could hemophagocytic lymphohistiocytosis be the core issue of severe COVID-19 cases?. BMC Med. 2020;18(1):214. Published 2020 Jul 15. doi:10.1186/s12916-020-01682-y

5. Ramos-Casals M, Brito-Zerón P, López-Guillermo A, Khamashta MA, Bosch X. Adult haemophagocytic syndrome. Lancet. 2014;383(9927):1503-1516. 10.1016/S0140-6736(13)61048-X

6. Otsuka R, Seino KI. Macrophage activation syndrome and COVID-19. Inflamm Regen. 2020;40:19. Published 2020 Aug 6. doi:10.1186/s41232-020-00131-w

7. Crayne CB, Albeituni S, Nichols KE, Cron RQ. The immunology of macrophage activation syndrome. Front Immunol. 2019;10(FEB):1-11.

8. Ferrara JL, Abhyankar S, Gilliland DG. 1993. Cytokine storm of graft-versus-host disease: a critical effector role for interleukin-1. Transplant. Proc. 25:1216-1217

9. Tisoncik JR, Korth MJ, Simmons CP, Farrar J, Martin TR, Katze MG. Into the eye of the cytokine storm. Microbiol Mol Biol Rev. 2012;76(1):16-32. doi:10.1128/MMBR.05015-11

10. Hussell T, Goulding J. 2010. Structured regulation of inflammation during respiratory viral infection. Lancet Infect. Dis. 10:360-366

11. La Gruta NL, Kedzierska K, Stambas J, Doherty PC. 2007. A question of self-preservation: immunopathology in influenza virus infection. Immunol. Cell Biol. 85:85-92

12. Simmons CP, et al. 2007. Patterns of host genome-wide gene transcript abundance in the peripheral blood of patients with acute dengue hemorrhagic fever. J. Infect. Dis. 195:1097-1107

13. Simmons CP, et al. 2006. Pretreatment intracerebral and peripheral blood immune responses in Vietnamese adults with tuberculous meningitis: diagnostic value and relationship to disease severity and outcome. J. Immunol. 176:2007-2014

14. Levy MM, et al. 2003. 2001 SCCM/ESICM/ACCP/ATS/SIS International Sepsis Definitions Conference. Crit. Care Med. $31: 1250-1256$

15. Lee SM, et al. 2010. Systems-level comparison of host responses induced by pandemic and seasonal influenza A H1N1 viruses in primary human type I-like alveolar epithelial cells in vitro. Respir. Res. 11:147.

16. Shinya K, et al. 2006. Avian flu: influenza virus receptors in the human airway. Nature 440:435-436

17. Guo XJ, Thomas PG. New fronts emerge in the influenza cytokine storm. Semin Immunopathol. 2017;39(5):541-550. doi:10.1007/s00281-017-0636-y

18. $\mathrm{Hu} \mathrm{B}$, Huang S, Yin L. The cytokine storm and COVID-19 [published online ahead of print, 2020 Jun 27]. J Med Virol. 2020;10.1002/jmv.26232. doi:10.1002/jmv.26232 
19. Hussman JP. Cellular and molecular pathways of COVID-19 and potential points of therapeutic intervention.OSF Preprints 2020, May 19 [Available from: https://doi.org/10.31219/osf.io/p69g8.

20. Haiming W, Xiaoling X, Yonggang Z, et al. Aberrant pathogenic GM-CSF+ T cells and inflammatory CD14+CD16+ monocytes in severe pulmonary syndrome patients of a new coronavirus. BioRXiv. 2020.

21. Zuo Y, Yalavarthi S, Shi H, et al. Neutrophil extracellular traps in COVID-19. JCI insight. 2020

22. Yao XH, Li TY et al A pathological report of three COVID-19 cases by minimally invasive autopsies. Chin. J. Pathol 2020; 49: E009.

23. Abraham E, Wunderink R, Silverman H, Perl TM, Nasraway S, Levy H, et al. Efficacy and safety of monoclonal antibody to human tumor necrosis factor $\alpha$ in patients with sepsis syndrome: a randomized, controlled, double-blind, multicenter clinical trial. JAMA 1995; 273: 934-41.

24. Bate R, Coticelli P, Tren R, Attaran A. 2008. Antimalarial drug quality in the most severely malarious parts of Africa - a six country study. PLoS One 3:e2132.
25. Brun-Buisson C, Richard JC, Mercat A, Thiebaut AC, Brochard L. 2011. Early corticosteroids in severe influenza A/H1N1 pneumonia and acute respiratory distress syndrome. Am. J. Respir. Crit. Care Med. 183:1200-1206

26. Meijvis SC, et al. 2011. Dexamethasone and length of hospital stay in patients with community-acquired pneumonia: a randomised, double-blind, placebo-controlled trial. Lancet 377:2023-2030

27. RECOVERY Collaborative Group, Horby P, Lim WS, et al. Dexamethasone in Hospitalized Patients with Covid-19 Preliminary Report [published online ahead of print, 2020 Jul 17]. $N$ Engl J Med. 2020;NEJMoa2021436. doi:10.1056/NEJMoa2021436

28. Tomazini BM, Maia IS, Cavalcanti AB, et al. Effect of Dexamethasone on Days Alive and Ventilator-Free in Patients With Moderate or Severe Acute Respiratory Distress Syndrome and COVID-19: The CoDEX Randomized Clinical Trial [published online ahead of print, 2020 Sep 2]. JAMA. 2020;10.1001/jama.2020.17021. doi:10.1001/jama.2020.17021 